PROKLA-Redaktion

\section{Editorial: Sozialismus?}

Vom 21. bis 26. Oktober 1985 fand in Cavtat bei Dubrovnik das zehnte „round table“ Gespräch der Zeitschrift „Sozialismus in der Welt ${ }^{\text {"s }}$ statt. Das Thema jener Vetanstaltung war Sozialismus in 21. Jahrhundert". In der intellektuell recht offenen Atmosphäre Jugoslawiens ging es darum, sich Rechenschaft übe neue Technologien, die sozialen Bewregungen, über die damalige Krise des Kapitalismus und die Möglichkeiten des Sozialismus vor der Schwelle des neuen Jahrtausends abzulegen. Fünf Jahre später schien es kaum noch möglich, von einer sozialistischen Zukunft zu sprechen. Dic "Volksdemokratien", in denen kommunistische Parteien regiert hatten, waren ebenso wie die Sowjetunion am Ende Auch jener Teil der Linken in den westlichen Ländern, die den "Realsozialismus schon lange kritisiert hatten, waren angesichts von dessen Zusammenbruch weitgehend sprachlos. Wer an sozialistischen Vorstellungen festhielt, tat dies eher defensiv (vgl. die Beiträge in PROKLA 78 Auf der Suche nach dem verlorenen Sozialismus, März 1990). Markt und repräsentative Demokratie triumphierten, wieder einmal redeten die euphorisierten bürgerlichen Geschichtsphilosophen vom Ende der Geschichte.

Doch obwohl diese Jahre eine Zeit ohne Alternative zu sein schienen, war dem nicht so. Zur Weltumweltkonferenz 1992 in Rio de Janeiro konnten Basisbewegungen aus allen Regionen der Erde im umfassenden Sinn globale Themen auf die Agenda der Weltpolitik bringen. In Mexiko begann der Widerstand der $\mathrm{Za}$ patisten, auf dem lateinamerikanischen
Kontinent kamen mehr oder weniger linke Regierungen zustande, es begann die Serie der Weltsozialforen. Die Kämpfe der indigenen Bevölkerung, die Kämpfe der Landlosen, die Fabrikbesetzungen ließen erkennen, dass der Neoliberalismus nicht damit erfolgreich war, die Zukunft unter seinen monokulturellen Litaneien zu begraben. Das 150 jährige Jubiläum des „Kommunistischen Manifests im Jahre 1998 zog erstaunlich große Aufmerksamkeit auf sich, der Erfolg, der Hardt/Negris "Empire“ beschieden war, zeigte dass ein enormes Bedürfnis nach

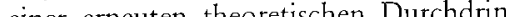
gung des Kapitalismus existierte, insbesondere bei all jenen, die die formellen und informellen Treffen der Regierenden begleiteten und gegen die neoliberalen Strategien der Aneignung und Enteignung kämpften. Die Enttäuschung über die eingeschränkten Möglichkeiten der offiziellen Politik, die Verzweiflung über die Realitätsverleugnung der Herrschenden veranlasste manchen, der mit de Linken gebrochen hatte, sich wieder an deren Existenz zu erinnern. Das Projekt des Sozialismus fand unter anderem Ausdruck in der Forderung von Hugo Chávez nach einem „Sozialismus des 21. Jahrhunderts" oder in den Reflexionen des italienischen Philosophen und früheren Abgeordneten des Europäischen Parlaments Gianni Vattimo darüber, warum er wieder zum Kommunisten wurde

Ausgerechnet in Deutschland scheint sich nun aus jener Nachfolgepartei der SED, die lange Zeit als Organisation von Altkommunisten und DDR-Seilschaften verdächtigt wurde, und aus verschiede- nen Gruppen und Einzelnen aus dem linken Spektrum Westdeutschlands, Vertretern der Gewerkschaften und von Arbeitslosigkeit und Verelendung Betroffenen ein neues linkes Projekt $z u$ formieren, das einen weit größeren gesellschaftlichen Rückhalt entwickelt, als in den vergangenen Jahrzehnten. War "Sozialismus" über Jahre ein Unwort, da öfentlich nicht in einem positiven Sinne verwendet werden konnte, wollte man sich nicht lächerlich machen, stellt es inzwischen wieder einen Bezugspunkt de wissenschaftlichen und politischen Diskussion dar. Selbst im deutschen Fernsehen wird hin und wieder die Frage nach Alternativen zum Kapitalismus aufgeworfen. Auch in anderen europäischen Ländern, in denen mit dem Scheitern der kommunistischen Parteien, dem Wechse der Sozialdemokratie in die neue Mitt der Gesellschaft und der Selbsteingliederung des grünen Projekts in die ökologi sche Modernisierung des Kapitalismus die Linke in die Krise geriet sind neue linke Parteien entstanden, und die sozialistische Diskussion lebte wieder auf. Allerdings macht die Krise der italienischen Rifondazione auch deutlich, dass allzu hochfliegende Hoffnungen auf neue Parteiprojekte sehr schnell enttäuscht werden können.

Sozialismus ist die Alternative zum Kapitalismus. Das ist keine Verkündigung und keine Offenbarung. Die Vorstellung, mit dem Fall der Mauer, dem Ende der Sowjetunion und der DDR sei das Projekt des Sozialismus zu Ende, ist kurz sichtig und unhistorisch. Der Sozialismus hat sich mit der Gesellschaftsformation, in der die kapitalistische Produktionsweise herrscht, herausgebildet. Er gehört zu ihr, ist ein Teil von ihr und gleichzeitig ist er über sie schon hinaus.

So speist sich der Sozialismus unter anderem aus den Forderungen nach Gerechtigkeit, aus den bürgerlichen Normen der Gleichheit, der Freiheit und der Solidarität. Insofern ist der Sozialismu im Innern der bürgerlichen Gesellschaf angesiedelt. Sozialismus ist der energische
Teil der bürgerlichen Aufklärung. Gleichheit und Freiheit sollen mehr sein als nur eine regulative Idee, der wir uns vielleicht immer annähern, um sie doch nie zu erreichen; diese Normen sollen die gegenständliche Welt bestimmen, objektive Wirklichkeit werden. Der Sozialismus kann gerade wegen dieser Zugehörigkeit zur bürgerlichen Gesellschaft selbst aber auch zu brav, zu harmlos und zu unaufgeklärt sein. So war der Sozialismus im Selbstverständnis seiner Vertreter oftmals nicht mehr als eben die Verwirklichung dieses immanenten Potentials der bürgerlichen Welt, die Verwirklichung ihrer Wünsche und Hoffnungen, ihre Ausdehnung auf alle.

Indem der Sozialismus aber auf die $\mathrm{Ab}$ schaffung aller Herrschaftsverhältnisse abzielt, nicht nur der persönlichen Herrschaftsverhältnisse, die vom Bürgertum angegriffen wurden, als dieses, gestütz auf den "Vierten Stand", an die staatliche Macht gelangte, sondern auch der unpersönlichen Herrschaftsverhältnisse, den stummen Zwang der ökonomischen Verhältnisse" (MEW 23: 765), der die bürgerliche Form der Herrschaft verewigt, geht der Sozialismus weit über die Realisierung bürgerlicher Normen hinaus.

Der Sozialismus ist ein Projekt, das sich über sich und seine Schwächen aufklärt, das gleichzeitig mit der Forderung nach der Verwirklichung der bürgerlichen Normen von diesen immer auch kritisch Abstand genommen hat. Das hat ihm den Vorwurf eingebracht, ein bloß taktisches Verhältnis zu den Gleichheits- und Freiheitsidealen oder zu den demokratischen Mitspracherechten zu haben. Aber der Sozialismus greift kritisch auch über die Formen der Produktion, der Verteilung, der Politik, der Beteiligung, der Le bensweise, die die bürgerliche Gesellschaft zu bieten hat, hinaus. Denn die Normen der Freiheit und Gleichheit sind vielfach schon verwirklicht. Diese Normen könnten immer weiter und tiefer verwirklicht werden und würden doch den entscheidenden qualitativen Ein- 
schnitt nicht mit sich bringen, der möglich und notwendig ist. Bei diesem Einschnitt handelt es sich darum, die Gesellschaft um ihr heimliches Zentrum herum zu organisieren, um die gesellschaftliche Arbeit selbst, um die Orte in den Fabriken, Büros und Familien, in denen de Reichtum der Gesellschaft erzeugt wird. Es handelt sich darum, die politische Ökonomie des Kapitals durch die politische Ökonomie der Arbeit zu ersetzen, gleichzeitig die Geschlechterverhältnisse fundamental zu verändern und jede Form rassistischer Diskriminierung zu beseitigen. Die Lasten der notwendigen Arbeit auf alle zu verteilen und $\mathrm{zu}$ ermöglichen, dass alle gleichermaßen ohne Sorge ums tägliche Überleben, ohne ständig clever sein zu müssen, entspann in den Genuss der gesellschaftlichen Freiheit gelangen, fröhlich mit den anderen leben und arbeiten, gemeinsam das Gemeinsame erzeugen, von den Gegenständen bis zu den Verhältnissen, unter denen sie leben, und ansonsten nicht tun wie ein Tier, ,auf dem Wasser liegen und friedlich in den Himmel schauen (Adorno)

Die kapitalistische Produktionsweise hat einen ungeheuren Reichtum erzeugt. Doch er beruht auf Ausbeutung und $\mathrm{Na}$ turzerstörung. Der Kapitalismus kann seine eigenen Voraussetzungen nicht reproduzieren. Regelmäßig gerät er in größere und kleinere Krisen. Arbeitslosigkeit und Schufterei, größter privater Luxus und das Uberleben auf niedrigstem $\mathrm{N}$ veau, Überfluss und Hunger, Freiheit und Folter, urbanes Leben und Vertrei bung, die Degradierung und Zerstörung der Umwelt sind unauflöslich durch di Gesetzmäßigkeiten dieser Gesellschaftsformation direkt miteinander verbunden. Es handelt sich nicht um unerwünschte Nebenfolgen. Sie würden sich auch nicht in Luft auflösen, wenn - wie manche hoffen - die Märlkte wieder verstärk staatlich reguliert und sozial eingebettet würden. Auch die schon von Engels kritisierte "Verstaatlichungssucht" (MEW 35: 427) von so manchen Linken wird hier nicht weiter helfen. Die Logik de kapitalistischen Produktionsweise selbs ist das Problem. Diese Logik verleiht der bürgerlichen Gesellschaftsformation den Charakter von Natur. Ihre Gesetzmäßigkeiten wirken wie Naturgesetze. Ein guter Teil der Politik, der so genannten öffentlichen Diskussion, der Bildungsprozesse besteht darin, die Bereitschaft auszubilden, sich diesen gesellschaftlichen Naturzwängen zu unterwerfen und das Sapere Aude! der Aufklärung zurückzunehmen, den Willen zur Emanzipation von der selbstverschuldeten Unmündigkeit.

Die Naturgesetze des Kapitals führen ständig und immer wieder zu Krisen, zu Arbeitslosigkeit, zu Hunger, Mord und Totschlag. Das kann gelegentlich vergessen gehen. Es können Hoffnungen aufkeimen und genährt werden, es ließen sich unter kapitalistischen Verhältnissen die Opfer vermeiden, die Ausbeutung auf ein akzeptables Maß verringern. Das mag hier und da, für eine gewisse Zeit möglich sein, aber im Durchschnitt der kapitalistischen Verhältnisse ist dies nicht der Fall

Doch auch die Normalität des Kapitalismus, nicht erst seine Krise, beruht auf Ausbeutung, auf der Unterordnung des gesellschaftlichen Lebens, wie des Lebens der Mehrzahl der Einzelnen unter die Verwertungszwänge des Kapitals. Nur wenn sich meine Arbeit für das Kapital rentiert, kann ich mit Lohn rechnen, nu was Profit erwarten lässt, wird produziert. Immer stärker werden nicht nur die Systeme sozialer Sicherung sondern auch die Bildungs- und Ausbildungsinstitutionen den Erfordernissen der Kapitalverwertung unterworfen.

Es bedarf der grundlegenden Veränderung der Produktionsverhältnisse, nicht allein dieser, das versteht sich, aber eben auch dieser. Eine solche Veränderung is eine Voraussetzung dafür, dass sich auch in anderen Bereichen Grundlegendes ändern kann. Was bislang als Armut erlebt wurde, Arbeitslosigkeit und Kurzarbeit kann sich in Zeitwohlstand verwandeln. Die freie Zeit kann für die gemeinsamen
Angelegenheiten, für Bildung, für Kinder verwendet werden. Wenn der Kampf um Überleben, wenn die Gesetze der Konkurrenz außer Kraft gesetzt werden, mus nicht jede Lebenssekunde auf Karriereplanung verwendet werden, um nur keine Chance zu verpassen, der soziale Verkehr mit anderen wird freier. Die Familie kann den Charakter der privaten Akkumulationsmonade aufgeben, in der die Partne einen Vertrag für die wechselseitige Nutzung threr Geschlechtsorgane abschlieBen und sich wechselseitige Absicherung gewähren. Kinder werden nicht als privater kosten- und zeitaufwendiger Wettbewerbsnachteil und als Anlass regressive Sinnkompensation betrachtet, sondern als Moment der Freiheit aller, die dem gemeinsamen Leben die Zukunft geben. Das gesellschaftliche Verhältnis zur $\mathrm{Na}$ tur kann sich verändern, sie kann als ein Reichtum gesehen werden, der der Menschheit in all ihren Generationen gehört und zu der der Mensch als Gattungswesen selbst gehört.

Sozialismus ist, hervorgehend aus den sozialen Kämpfen in Europa, aber schon lange nicht mehr auf diese begrenzt, de Name für Emanzipation geworden. Dieser Name wurde durch die gescheiterten Versuche, sozialistische Verhältnisse einzurichten, diskreditiert. Viele Millionen Menschen wurden im Namen des Sozialismus getötet, ermordet, drangsaliert, um ihre Lebensmöglichkeiten gebracht. Die Menschen müssen, selbst wenn sie für den Sozialismus Sympathie haben, befürchten, bei jedem weiteren Versuch, ihn herzustellen, das wenige Gute, was sie haben, zu verlieren und etwas Schlechteres dafür zu erhalten. Trotz dieser zweifelhaften Geschichte haftet die Idee der Emanzipation am Begriff des Sozialismus. Er kann nicht aufgegeben werden, wenn unter kapitalistischen Bedingungen grundsätzliche Kritik laut wird und de Wunsch nach Alternativen zur kapitalistischen Vergesellschaftung entsteht. Es is keine neue Erkenntnis, aber sie gilt weiterhin, dass die Theorte und die Praxis, die im Namen des Sozialismus stattfand, der kritischen Untersuchung bedarf. Eine solche Forschung hat aus verständlichen Gründen schlechtere Bedingungen unter den kapitalistischen Verhältnissen als kritische Gegenwartsanalysen, da sie eine mögliche alternative Zukunft vorbereiten hilft. Sie ist aber vielleicht noch dringlicher als die genaue Kenntnis der Gesetzmäßßigkeiten der bürgerlichen Gesellschaftsformation. Denn nur wenn im Vorgriff auf das zukünftige Emanzipationspotential deutich wird, wie vermieden werden kann, dass sich von neuem autoritäre Kräfte durchsetzen, können die Individuen ihre Wünsche nach einem freien Leben und nach Gestaltung mit einer sozialistischen Zukunft verbinden. Nicht über diese Zukunft nachzudenken, hieße zu erwarten, dass sich der Übergang von der kapitalistischen zur sozialistischen Gesellschaft gleichsam automatisch vollzieht. Doch das schließt die Gefahr ein, politische Chancen nicht zu nutzen und vermeidbare Fehler - im günstigeren Fall als Farce - zu wiederholen.

Manche halten jede modellhafte Konzeptualisierung von Alternativen für im schlechten Sinne utopisch und plädieren dafür, die Klärung der Probleme einer sozialistischen Alternative der Zukunft zu überlassen. Bei einem linken „Bilderverbot" kann es freilich nicht bleiben, denn Mehrheiten für eine sozialistische Alternative können nur gewonnen werden, wenn deren Machbarkeit plausibel gemacht werden kann und von Vielen getragene Strategien der Transformation entwickelt werden. Dies gilt erst rech nach dem Scheitern des „real existierenden Sozialismus". Um nicht im schlechten Sinne utopisch zu sein, müssen Konzeptionen des Sozialismus von der Kritik am Kapitalismus und an den gescheiterten historischen Versuchen des Über gangs zum Sozialismus ausgehen.

Dabei können wir zuallererst von Marx lemen, der nicht nur die kapitalistische Produltionsweise, sondern auch die sozialistischen Strömungen seiner Zeit einer radikalen Kritik unterzogen hat. Aus dieser doppelten Kritik resultierte Marx 
Überzeugung, dass die Zukunft einer As soziation freier Individuen gehören müsse, die nur erreichbar ist, wenn die Arbeiterklasse sich selbst befreit. Daher lehnte Marx alle Versuche ab, den Sozialismus gleichsam von oben durch putschistische und autoritäre Strategien durchzusetzen Wie Hendrik Wallat in seiner Rekonstruktion der Marxschen Sozialismuskritik zeigt, war Marx gleichermaßen kritisch gegenüber dem Etatismus in der Arbeiterbewegung wie auch gegenüber dem "kleinbürgerlichen Sozialismus", der auf die Abschaffung bestimmter Kapital formen, aber die Beibehaltung der Marktwirtschaft setzte. Marx erwartete vielmehr, dass die Trennung von Politik und Ökonomie hinfällig wird, dass sowohl der Staat als auch der Markt absterben, wenn die Subalternen ihr Schicksal selbst in die Hand nehmen.

Die Marxschen Vorstellungen haben sich in der Arbeiterbewegung nur zum Teil durchgesetzt, wie Ralf Hoffrogge am Beispiel der Entwicklung der deutschen $\mathrm{Ar}_{\mathrm{r}}$ beiterbewegung zwischen 1848 und 1920 deutlich macht. Hier spielten zunächs die staatsgläubigen und erzieherischen Vorstellungen Ferdinand Lassalles eine wichtige Rolle. Nach ihrer „marxistischen Wende" dominierten in der deutschen Sozialdemokratie eine reformistische Praxis und eine verbalradikale, abwartende Haltung, die auf den automatischen Zusammenbruch des Kapitalismus in der Zukunft setzte. Erst die Krise des ersten Weltkriegs, die die Sozialdemolratie vor die Alternative zwischen nationalem Sozialismus und Weltrevolution stellte und zu ihrer Spaltung führte, brachte eine Erneuerung des Sozialismus in Theorie und Praxis mit sich. An die Erfahrungen der Rätebewegungen lässt sich heute noch anknüpfen.

Alex Demirovic macht in seinem Beitrag deutlich, dass die Konzepte der Rätedemokratie - von Marx Reflexionen über die Pariser Kommune bis zu den Theoretikern der Rätebewegung nach dem Ende des ersten Weltkriegs - trotz einer Reihe offener Fragen kohärente Ansatzpunkte für Emanzipationsbewegungen bilden: Die Rätedemokratie ist die politische Form, in der der Übergang zum Sozialismus organisiert werden kann.

Das Scheitern der ersten Versuche, den Sozialismus herzustellen, hängt wesentlich damit zusammen, dass die in den revolutionären Auseinandersetzungen spontan entstandenen Formen der Räte demokratie alsbald durch eine autoritäre Parteiherrschaft ersetzt wurden. Das Privateigentum an Produktionsmitteln wurde zwar durch ihre Verstaatlichung aufgehoben, aber die sich entwickelnde Zentralverwaltungswirtschaft bedeutete eben nicht, dass die assoziierten Produzenten sich die Produktionsmittel aneigneten. Vielmehr bestand eine Gemeinsamkeit zwischen dem "real existierenden Sozialismus" und dem Kapitalismus darin, dass die Trennung der unmittelbaren Produzenten von den Produktionsmitteln weiter existierte. Das „Volkseigentum" blieb, wie Renate Hürtgen in ihrem Beitrag über die Betriebe in der DDR darstellt, formal; die - in der Praxis häufig recht planlose - Planwirtschaft war nicht demokratisch und partizipativ organisiert, daher auch nicht an den Bedürfnissen der Individuen orientiert. Die Lage der Arbeiter in der DDR ähnelte, was Arbeitsorganisation, Leistungsbewertung und Entlohnung angeht, durchau der im „Westen“. Die realen Möglichkei ten der betrieblichen Mitbestimmung seitens der Arbeiter waren sehr beschränkt. Der Betrieb blieb Organisation von Herrschaft über Menschen.

Bis heute gibt es selbst in der marxistischen Diskussion keinen Konsens darüber, wie die Sowjetunion und die ih ähnlichen Gesellschaften, die mit dem Anspruch auftraten "sozialistisch" zu sein, auf den Begriff zu bringen sind Marcel van der Linden gibt einen Überblick über die marxistischen Kritiken des "real existierenden Sozialismus". Er unterscheidet die von Trotzki herrührende Theorie des degenerierten Arbeiterstaates, Theorien des bürokratischen Kollektivismus und Theorien des Staatskapita- lismus. Er betont vor allem die Bedeutung der Weltmarktverhältnisse für die Entwicklung der Sowjetgesellschaft und sieht den Stalinismus als eine Form der „ursprünglichen Akkumulation", deren Dynamik eher indirekt, von außen bestimmt wurde.

Raul Zelik dagegen betont in seinem Beitrag stärker die Widersprüche in den realsozialistischen Gesellschaften sowie die systemischen Probleme der Ressourcenallokation und Innovation in Planwirtschaften, um zu erklären, warum der Staatssozialismus im Hinblick auf Effizienz und Nachhaltigkeit letztlich noch schlechter abschnitt als der Kapitalismus. Die Vergesellschaftung war demnach nur vordergründig durch die Planung bestimmt; die sozialen Konflikte artikulierten sich hinter der Erscheinungsebene des Plans. In ihren Zielsetzungen blieb die Planung zudem stark der kapitalistischen Akkumulationslogik verhaftet.

Nach dem Zusammenbruch der zentral gelenkten Planwirtschaften waren viele Linke, sofern sie nicht ohnehin von sozialistischen Zielen Abschied nahmen, der Ansicht, dass allenfalls der „Marktsozialismus" eine gangbare Alternative zum Kapitalismus wäre. Schon die Reformversuche innerhalb des "real existierenden Sozialismus“, von der "neuen ökonomischen Politik" unter Lenin über die Reformen in Jugoslawien und Ungarn, das "neue ökonomische System der Planung und Leitung" in der DDR und die Reformen des "Prager Frühlings" bis zur „Perestroika " unter Gorbatschow, hatten in der Regel darauf gezielt, Marktelemente zu stärken und mit der zentralen $\mathrm{Pla}$ nung zu verbinden. Heute beruft sich die Kommunistische Partei Chinas auf das Konzept einer „sozialistischen Marktwirtschaft" und entwickelt kapitalistische Verhältnisse (vgl. dazu PROKLA 119 Chinesischer Kapitalismus, Juni 2000). Katharina Götsch setzt sich kritisch mit den Konzeptionen des Marktsozialismus auseinander. Gestützt auf die Marxsche Kritik an Proudhon, die Analyse de marktwirtschaftlichen Reformen in den
Ländern des ,real existierenden Sozialismus" und die Kritik an neueren marktsozialistischen Modellen wie dem von John Roemer kommt sie - anders als etwa Raul Zelik - zu dem Ergebnis, dass Markt und Sozialismus im Prinzip unvereinbar sind. Die Alternative zur gescheiterten Zentralverwaltungswirtschaft ist demnach nicht die sozialistische Marktwirtschaft, sondern die Selbstregierung der Produzenten und Konsumenten mittels einer demokratischen, partizipativen Planung. Nicht die Planwirtschaft als solche ist gescheitert, sondern ihre autoritäre Variante.

Marx behauptete, dass eine Gesellschaftsformation nie untergeht, "bevor alle Produktivkräfte entwickelt sind, für die sie weit genug ist ${ }^{\varsigma}$, und dass „höhere Produktionsverhältnisse" nie an ihre Stelle treten, „bevor die materiellen Existenzbedingungen derselben im Schoß der atten Gesellschaft selbst ausgebrütet worden sind" (MEW 13: 9). Wie neue Produktivkräfte mit den kapitalistischen Produktionsverhältnissen in Konflikt geraten, lässt sich gegenwärtig etwa im Bereich der freien Softwareproduktion und der Verteilung digitaler Güter über das Internet beobachten. Nur mühsam gelingt es (wenn überhaupt), mittels der "geistigen Eigentumsrechte" und des Staates, offenbar obsolete Formen der privaten, kapitalistischen Aneignung des produzierten Reichtums weiter zu gewährleisten. Christian Siefkes sieht in der "Commonsbasierten Peer-Produktion" im Bereich der Informationsproduktion und der Informationstechnik bereits den Keim einer neuen Produktionsweise, die kommunistischen Zielen nahe kommt. Wir hoffen in diesem Sinne, dass die PROKLA dazu beiträgt, dass die Menschheit sich endlich jene Aufgaben bewusst stellt, für deren Lösung die materiellen Bedingungen bereits vorhanden sind. An dieser Ausgabe wirkten Ingo Stützle und Raul Zelik als Gastredakteure mit Für ihre vielfältigen Anregungen und ihr Engagement sei ihnen ganz herzlich gedankt. 
$* * *$

Zur PROKLA 154 Mit Steuern steuern ist noch nachzutragen, dass der Text von Michael Krätke „Kritik der öffentlichen

Finanzen. Die Finanzkrise des Staates er-

\section{PROKLA 156: Ökologie in der Krise (September 2009)}

Mit dem Ausbruch der Finanzkrise wurde die Verzahnung von ökonomischer und ökologischer Krise schnell deutlich. Einerseits wird befürchtet, dass insbesondere der Klimaschutz den diversen Konjunkturpaketen zum Opfer fällt und ein Ausstieg aus dem fossilen Kapitalismus in weite Ferne rückt, andererseits wird die ökonomische Krise aber auch als Chance gesehen, um im Rahmen eines „Green New Deal“ nun endlich die drängenden ökologischen Probleme anzugehen. Schon vor Jahrzehnten war auf die „ökologischen Grenzen des Wachstums“ hingewiesen worden. Daraus wurde dann vielfach der Schluss gezogen, dass die kapitalistische Produktionsweise mit ih rem Wachstumsimperativ keine adäquate Lösung der ökologischen Krise finden könne und folglich, angesichts der Endlichkeit der Ressourcen, an immanente, stoffliche Grenzen stoßen müsse. Doch sind schon seit längerer Zeit Veränderungen von Betriebsweisen und Unternehmensstrategien zu beobachten; in jüngster Zeit wird, teils kritisch, teils affirmativ, von einem "Grünen Kapitalismus" gesprochen. Die PROKLA möchte die grüne Formierung des Gegenwartskapitalismus im Kontext der ökonomischen Krise beleuchten. Welche strukturellen Veränderungen zeigen sich in der Organisation der kapitalistischen Produktionsweise, welche neuen Ausschlussmechanismen ergeben sich aufgrund der ölologischen Modernisierung des Kapitalismus, welche Konsequenzen haben diese Prozesse für soziale Bewegungen?

\section{PROKLA 157 Der Blutige Ernst: Krise und Politik (Dezember 2009)}

Was hierzulande als konjunktureller Abschwung und in den USA als Immobilienkrise begann, ist dabei sich zu einer Weltwirtschaftskrise zu entwickeln, deren ganzes Ausmaß noch immer nicht abzuschätzen ist. Tiefgreifende Krisen sind üblicherweise Phasen, in denen sich die jeweilige historische Gestalt der kapitalistischen Produktionsweise verändert. Bislang war es vor allem die staatliche Politik, die durch die Krise eine Aufwertung erfahren hat. Galt innerhalb des neoliberalen Diskurses, der in den letzten Jahren vorherrschte, die staatliche Politik gleichermaßen als unfähig (da der Markt alles besser könne) und ohnmächtig (angesichts der Globalisierung bliebe den Nationalstaaten sowieso keine Eingriffsmöglichkeiten mehr), so wird nun von allen Seiten nach dem Staat als Retter gerufen. Wird sich dadurch das Verhältnis von Politik und Ökonomie nachhaltig verändern? Werden wir erneut einen „regulierten“ Kapitalismus erleben? Und wem nützt er? Welche Klassen(fraktionen) werden zu den Gewinnern bzw. den Verlierern der Krise gehören? Die Kosten der Krisenbewältigung werden gewaltig sein, aber wer wird sie zu tragen haben? Die Krise hat auch eine internationale Dimension, sie wird die relative Stärke der großen kapitalistischen Ökonomien verändern, auch hier wird es Gewinner und Verlierer geben. Welche weltwirtschaftlichen, aber auch weltpolitischen Verschiebungen, welche Konfliktlinien zeichnen sich ab?

\section{Alex Demirović}

\section{Rätedemokratie oder das Ende der Politik}

\section{Das Versagen der liberalen Demokratie}

In der Novemberrevolution, deren 90jähriges Jubiläum dieses Jahr gefeiert wird, konnten die Linke und die deutsche Gesellschaft wählen zwischen Sozialismus, der auf die Einheit von Politik und Produktionsverhältnissen in Gestalt einer Rätedemokratie zielte, und Sozialdemokratie, die auf die Herstellung der parlamentarischen, repräsentativen, liberalen Demokratie zielte, die die Emanzipation in der Durchsetzung formaler Gleichheits- und Freiheitsrechte, also in der Überwindung der Feudalität und der Herstellung der Trennung von Markt und politischer Sphäre sah. Das Scheitern der sozialistischen Revolution in Deutschland gab gesellschaftlichen Entwicklungstendenzen Raum, die zu enormer Kapitalmacht führten und massgeblich zu einer autoritären Lösung der Weltwirtschaftskrise nach 1929 beitrugen. Die liberale, repräsentative Demokratie konnte solchen autoritären Tendenzen nicht nur nichts entgegensetzen, vielmehr wurde aus der Mitte des demokratisch gewählten Parlaments heraus und von den bürgerlichen Parteien getragen die Entscheidung für einen von den Nationalsozialisten regierten autoritären Ausnahmestaat getroffen. Wie wenig geeignet die liberale Demokratie ist, autoritären Tendenzen entgegenzutreten, wurde in den vergangenen Jahren von der US-Regierung unter George W. Bush demonstriert. Aber es sind nicht nur die USA, für die eine Schwächung, wenn nicht sogar Aushöhlung der parlamentarischen Demokratie beobachtet wurde. Wieder einmal wird dies auch in Deutschland beobachtet.

„Wirtschaftsführer wie Politiker schwärmen zunehmend offen vom Can-do-Spirit des autoritären Lagers. Während sie in heimischen Landen vorwiegend Stagnation ausmachen, sehen sie anderswo nur den Boom und bewundern ihn oft kritiklos. ... Stimmen also unsere jahrzehntelang gehegten und gepflegten Grundannahmen nicht mehr, etwa jener berühmte Spruch von Winston Churchill, dass die Demokratie zwar unvollkommen und kritikwürdig, aber doch eindeutig das am wenigsten schlechte aller schlechten Regierungssysteme sei? Sind Gewaltenteilung und Menschenrechte eben kein universales Erfolgsmodell - funktionieren repressive Systeme womöglich am besten, kurze Wege statt langer Diskussionen, Sagen-wo's-langgeht statt Kompromissen?" (Der Spiege1, Nr. 19, 5.5.2008)

Was der "Spiegel" hier beschreibt, ist in gewisser Weise selbst schon überholt. Colin Crouch stellt fest, dass die parlamentarische Demokratie nicht mehr funktioniert, sondern in das Stadium der Postdemokratie übergegangen ist: die Fassade der Wahlen und des Parlaments funktioniert, aber die politischen Pro- 